\title{
Paradigm Shift in Anti-Coagulating Patients with Non-Valvular Atrial Fibrillation
}

\author{
Bushra Moiz
}

Non-valvular atrial fibrillation (AF) is the most frequent cardiac arrhythmia in aging population. It was estimated that 2.3 million US adults had AF in 2001, extrapolated to 2.5 -fold increase by $2050 .{ }^{1}$ AF produces turbulence of blood and endothelial injury in the left atrium (and its appendage) with consequential arterial thromboembolism. Clinically, the most significant event is the possibility of ischemic stroke (IS) as well as peripheral thrombi. Therefore, patients with AF irrespective of whether it is paroxysmal, persistent or permanent, require lifelong thromboprophylaxis. Anticoagulation reduces the risk of IS and other peripheral thrombi by approximately two-thirds of the baseline risks. Additionally, it not only decreases the severity of IS but it also decreases the 30-day mortality following stroke, if it does occur.

Given that anticoagulant therapy has both risks (principally bleeding) and benefits (reduced risk of thrombosis), many scoring systems have been described to estimate the risks of these outcomes. However, no single system is universally accepted or highly predictive. Gage et al. validated various classification schemes using clinical data of 1,733 patients, and concluded that $\mathrm{CHADS}_{2}$ scoring can predict the risk of IS in patients with AF. ${ }^{2}$ In $\mathrm{CHADS}_{2}, 1$ point is assigned each for the presence of congestive heart failure, hypertension, age 75 years or older, and diabetes mellitus; and 2 points for the history of stroke or transient ischemic attack. They reported that the risk of stroke per 100 patient-years (without anticoagulation) increased by 1.5 -fold for each point increase in $\mathrm{CHADS}_{2}$. In 2010, $\mathrm{CHADS}_{2}$, was further refined to $\mathrm{CHA}_{2} \mathrm{DS}_{2}$-Vasc by adding additional risk factors like female gender (1 point) and vascular disease (1 point for history of myocardial infarction, peripheral artery disease) and assigning 2 points to age $>75$ years instead of one-point in original scheme. ${ }^{3}$ As anticoagulating a patient will increase his bleeding risk; therefore, hemorrhagic risk assessment is also done. However, it is not clear that to what extent this scoring

Department of Pathology and Laboratory Medicine,

The Aga Khan University, Karachi, Pakistan

Correspondence: Prof. Bushra Moiz, Department of Pathology and Laboratory Medicine, The Aga Khan University,

Stadium Road, Karachi, Pakistan

E-mail: bushra.moiz@aku.edu

Received: October 01, 2018; Accepted: October 11, 2018 will affect decision-making during anti-coagulation of patients with AF. Apostolakis et al. compared various bleeding-risk prediction scores in AF and concluded that HAS-BLED score can estimate the risk of bleeding, particularly intracranial bleeding in patients taking anticoagulation. ${ }^{4}$ In HAS-BLED, one point each is assigned to hypertension, abnormal renal or hepatic function, stroke, bleeding, labile INR, elderly (>65 years) and drug or alcohol intake. Accordingly, an AF patient having a HAS-BLED score of $\geq 3$ is at increased risk of bleeding. American College of Chest Physicians (ACCP) guidelines recommend thrombo-prophylaxis with oral anticoagulation for patients with $\mathrm{AF}$ who are at high risk of stroke $\left(\mathrm{CHADS}_{2} \geq 2\right)$, while those having lower risk need personalised approach for AF management. 5 Similarly, guidelines are given by European Society of Cardiology, American College of Cardiology Foundation, and American Heart Association for anti-coagulating patients with AF.

Traditionally, oral vitamin $\mathrm{K}$ antagonist (VKA), e.g. warfarin, has been used to anti-coagulate patients with AF. However, this medication requires continuous laboratory monitoring to achieve a target of 2-3 of international normalised ratio, and has significant drugdrug, drug-disease and drug-food interaction. Moreover, intra-cerebral bleeding is the most dreadful complication of VKA toxicity. New oral anticoagulants (NOACs) revolutionise the prophylaxis and treatment of thrombotic disorders as they are taken in fixed doses, do not routinely require laboratory monitoring, and do not have drug-, food-, or disease interactions. Food and Drug Administration (FDA) authority approved NOACs for thromboprophylaxis in AF including direct thrombin inhibitor (dabigatran in 2010) and anti-Xa inhibitors (rivaroxaban in 2011, apixaban in 2012 and edoxaban in 2015). A meta-analysis, comparing safety and efficacy of NOACs with warfarin in over 44,000 patients, reported that NOACs were more efficacious than warfarin in preventing stroke and systemic embolism, and have lower risk of intracranial bleeding in patients with AF. 6 However, these results have been challenged recently because of lack of robustness in these clinical trials. ${ }^{7}$ In contrast to VKA that acts by inhibiting hepatic synthesis of vitamin K-dependent clotting factors (II, VII, IX and X), NOACs function by inhibiting active forms of clotting factors like Ila (by dabigatran) and $\mathrm{Xa}$ (by anti-Xa inhibitors). Bioavailability of NOACs ranges from $6 \%$ for 
dabigatran to $80 \%$ for rivaroxaban and apixaban. Their peak action starts within 1-3 hours (in contrast to 48-72 hours for VKAs) and are dependent on renal clearance for elimination.

One of the barriers in using NOACs is high prescription cost when compared with VKA. Conversely, recent reports from European countries have shown cost effectiveness of NOACs with improvement in qualityadjusted life years. Secondly, a dose adjustment is required for patients with renal failure as NOACs are mainly dependent on renal function for their clearance. Thirdly, NOACs till very recently had no antidotes for bleeding or surgical patients who require immediate reversal of anticoagulation. Hence, such patients were managed with supportive care on similar lines as a patient who is bleeding secondary to VKA toxicity. Recently, FDA-approved direct reversal agents (idarucizumab for dabigatran and andexanet alfa for FXa inhibitors) have been launched that may be useful for fast reversal of NOACs as compared to previously used reversal agents. 8 Finally, laboratory monitoring of NOACs is problematic. Though patients receiving NOACs are not routinely monitored, but laboratory testing becomes important when such a patient starts bleeding or requires surgery. Conventional prothrombin time (PT) and activated partial thromboplastin time (APTT) provide only qualitative assessment or limited information for NOACs. Therefore, while normal PT and APTT exclude the presence of clinically important drug effect, abnormal tests indicate that anticoagulation effect of NOACs is present. Thrombin time or diluted thrombin time for dabigatran and validated anti-Xa assay for rivaroxaban and apixaban can be used for monitoring.

Besides the limitations discussed above, patients with AF who were switched from VKA to NOACs reported more frequent bruising and depression/anxiety. ${ }^{9}$

In Pakistan, we do not know the true quantum of disease burden as well as type and frequency of treatment received. An insight was provided by a report from Ikramullah et al., who reported anticoagulation in 205 patients with AF at two tertiary care academic institutes. Of $149(73 \%)$ patients who were candidates of anticoagulation (according to risk stratification using $\mathrm{CHA}_{2} \mathrm{DS}_{2}$-Vasc), $27.5 \%$ received VKA or NOACs while remaining patients received either dual antiplatelet agents $(35 \%)$, single antiplatelet agent $(30 \%)$ or no treatment $(7 \%) .{ }^{10}$

This report does not describe details of NOACs prescription; however, it provides a glimpse of real life scenario in indigenous setting. There are patients-at- large in our communities who do not visit any doctor or die of IS secondary to AF before any medical help can be provided. The lack of local data necessitates large scale prospective studies for evaluating the true magnitude of AF, frequency of IS, type, compliance, cost effectiveness, and complications of anticoagulation as well as quality of life in patients having AF.

\section{REFERENCES}

1. Go AS, Hylek EM, Phillips KA. Prevalence of diagnosed atrial fibrillation in adults: national implications for rhythm management and stroke prevention: the anti-coagulation and risk factors in atrial fibrillation (ATRIA) study. JAMA 2001; 285:2370-5.

2. Gage BF, Waterman AD, Shannon W, Boechler M, Rich MW, Radford MJ. Validation of clinical classification schemes for predicting stroke: results from the national registry of atrial fibrillation. JAMA 2001; 285:2864-70.

3. Lip GY, Nieuwlaat R, Pisters R, Lane DA, Crijns HJ. Refining clinical risk stratification for predicting stroke and thromboembolism in atrial fibrillation using a novel risk factor-based approach: the euro heart survey on atrial fibrillation. Chest 2010; 137:263-72.

4. Apostolakis S, Lane DA, Guo Y, Buller H, Lip GY. Performance of the HEMORR(2) HAGES, ATRIA, and HAS-BLED bleeding risk-prediction scores in patients with atrial fibrillation undergoing anticoagulation: the AMADEUS (evaluating the use of SR34006 compared to warfarin or acenocoumarol in patients with atrial fibrillation) study. J Am Coll Cardiol 2012; 60:861-7.

5. You JJ, Singer DE, Howard PA. Antithrombotic therapy for atrial fibrillation. Chest 2012; 141:e531S-75S.

6. Miller CS, Grandi SM, Shimony A, Filion KB, Eisenberg MJ. Meta-analysis of efficacy and safety of new oral anticoagulants (dabigatran, rivaroxaban, apixaban) versus warfarin in patients with atrial fibrillation. Am J Cardiol 2012; 110:453-60.

7. Camm AJ, Fox KAA, Peterson E. Challenges in comparing the non-vitamin $\mathrm{K}$ antagonist oral anticoagulants for atrial fibrillation-related stroke prevention. Europace 2018; 20:1-11.

8. Udayachalerm S, Rattanasiri S, Angkananard T, Attia J, Sansanayudh N, Thakkinstian A. The reversal of bleeding caused by new oral anticoagulants (NOACs): A systematic review and meta-analysis. Clin Appl Thromb Hemost 2018: 1076029618796339

9. De Caterina R, Bruggenjurgen B, Darius H. Quality of life and patient satisfaction in patients with atrial fibrillation on stable vitamin $\mathrm{K}$ antagonist treatment or switched to a non-vitamin $\mathrm{K}$ antagonist oral anticoagulant during a 1-year follow-up: $\mathrm{A}$ PREFER in AF registry substudy. Arch Cardiovasc Dis 2018; 111:74-84

10. Ullah I, Ahmad F, Ahmad S, Hayat Y. Atrial fibrillation and stroke prevention practices in patients with candidacy for anticoagulation therapy. J Ayub Med Coll Abbottabad 2015; 27:669-72. 